\title{
Activated mTORC1 promotes long-term cone survival in retinitis pigmentosa mice
}

\author{
Aditya Venkatesh, ${ }^{1}$ Shan Ma, ${ }^{1,2}$ Yun Z. Le, ${ }^{3}$ Michael N. Hall, ${ }^{4}$ Markus A. Rüegg, ${ }^{4}$ and Claudio Punzo' \\ 'Department of Ophthalmology and Gene Therapy Center, University of Massachusetts Medical School, Worcester, Massachusetts, USA. ${ }^{2}$ Tianjin Medical University Eye Hospital, Nankai District Tianjin, China. \\ ${ }^{3}$ Department of Medicine, Section of Endocrinology, University of Oklahoma Health Sciences Center, Oklahoma City, Oklahoma, USA. “Biozentrum, University of Basel, Basel, Switzerland.
}

\begin{abstract}
Retinitis pigmentosa (RP) is an inherited photoreceptor degenerative disorder that results in blindness. The disease is often caused by mutations in genes that are specific to rod photoreceptors; however, blindness results from the secondary loss of cones by a still unknown mechanism. Here, we demonstrated that the mammalian target of rapamycin complex 1 (mTORC1) is required to slow the progression of cone death during disease and that constitutive activation of mTORC1 in cones is sufficient to maintain cone function and promote long-term cone survival. Activation of mTORC1 in cones enhanced glucose uptake, retention, and utilization, leading to increased levels of the key metabolite NADPH. Moreover, cone death was delayed in the absence of the NADPH-sensitive cell death protease caspase 2, supporting the contribution of reduced NADPH in promoting cone death. Constitutive activation of mTORC1 preserved cones in 2 mouse models of RP, suggesting that the secondary loss of cones is caused mainly by metabolic deficits and is independent of a specific rod-associated mutation. Together, the results of this study address a longstanding question in the field and suggest that activating mTORC1 in cones has therapeutic potential to prolong vision in RP.
\end{abstract}

\section{Introduction}

The interneuronal relationship between rod and cone photoreceptors in humans and mice is such that rod death always leads to cone death, while conversely, loss of cones has only a minimal effect on rods (1-4). This phenomenon plays a central role in retinitis pigmentosa $(\mathrm{RP})$, since mutations in a few rod photoreceptor-specific genes affect a disproportionately large number of patients (5) (RetNet: https://sph.uth.edu/retnet/). Because cones are essential for daylight, color, and high-acuity vision, it is their loss that leads to blindness. The fact that cone death always follows rod death independently of the underlying mutation in a rod-specific gene suggests that the reason(s) for cone death might be similar across various forms of RP. Thus, targeting the common mechanism(s) of cone death may allow for the development of vision therapies with broad clinical significance.

Many theories have been proposed to explain the dependence of cones on rods. Such theories include the production of toxic byproducts from dying rods, the loss of rod trophic support as rods die (6-11), overactive microglia recruited to remove the dying rods, and increased oxidative stress (12-16). We recently proposed a different theory suggesting that cones suffer from a nutrient shortage induced by disruption of the retinal architecture once most rods have been lost (17). Because rods outnumber cones at a greater than 20:1 ratio and photoreceptors account for approximately $75 \%$ of all retinal cells, loss of rods severely alters the retinal

\section{Related Commentary: p. 1390}

Authorship note: Aditya Venkatesh and Shan Ma contributed equally to this work. Conflict of interest: The authors have declared that no conflict of interest exists. Submitted: October 30, 2014; Accepted: February 5, 2015.

Reference information: J Clin Invest. 2015;125(4):1446-1458. doi:10.1172/JCI79766. architecture $(18,19)$. Thus, we hypothesized that once rod death progresses beyond a critical threshold, cone death initiates as a cell-autonomous event due to a reduction in nutrient flow from the adjacent cell layer, the retinal pigmented epithelium (RPE) (17). This hypothesis was based on the following findings: First, cone death always initiates only after approximately $90 \%$ of rods have died, irrespective of the rod death kinetics. Second, during the period of cone degeneration, cones display signs of prolonged starvation. Finally, during cone death, we observed gene expression changes in many metabolic genes and genes of the insulin/ mTOR pathway, a key pathway controlling cell metabolism (17). To test whether activation of the insulin/mTOR pathway alters cone survival, we treated the fast-progressing $r d 1$ mouse model (20) of RP with daily systemic injections of insulin (17). While cone survival did improve, the therapeutic effect of insulin lasted only for a period of 4 weeks, possibly due to the negative-feedback loop within the insulin/mTOR pathway (Figure 1 and refs. 17, 21). Our findings, though encouraging, left many questions unanswered regarding the role of $\mathrm{mTOR}$ and its potential as a therapeutic target to promote cone survival in RP. As such, it remained unclear whether insulin acts directly on cones to improve cone survival, or whether it stimulates other cells such as RPE cells or retinal Müller glia cells to release neuroprotective factors, as its administration was systemic. It was also unclear whether the protective effect of insulin requires mTOR activity and whether, by circumventing the feedback mechanism within the insulin/mTOR pathway, cone survival can be prolonged to the point of being therapeutically relevant to humans. Last, it remained to be tested whether the effect of insulin can be extended to other mouse models of RP, giving it a broader clinical significance.

To evaluate the long-term therapeutic potential of the insulin/mTOR pathway on cone survival and to test whether insulin 
acts directly on cones through this pathway, we have now constitutively activated this pathway in cones. Through the use of various conditional alleles of genes downstream of the insulin receptor (Figure 1) that were deleted using the same cone-specific Cre recombinase line (ref. 22 and Supplemental Figure 1, A-E; supplemental material available online with this article; doi:10.1172/ JCI79766DS1), we show that mTORC1 activity in cones is required to slow the progression of the disease and that constitutively active mTORC1 in cones is sufficient to significantly prolong cone survival and maintain cone function. This mechanism of protection functions in the fast-progressing $r d 1$ mouse model of RP and in the slow-progressing rhodopsin-KO (Rho-KO) (23) model. Activated mTORC1 increases the expression of genes that improve glucose uptake, retention, and utilization, resulting in increased NADPH levels. Because low NADPH levels can trigger cell death by activation of the initiator caspase, caspase 2 (CASP2) $(24,25)$, we crossed caspase 2 -deficient mice $\left(\operatorname{Casp}^{-/-}\right)$(26) with the fastprogressing $r d 1$ model of the disease, which slowed the progression of cone death. In summary, our results show that cell-autonomous activation of mTORC1 in cones is sufficient to promote long-term cone survival and that high glucose and NADPH levels are crucial for cone survival, indicating that the secondary loss of cones in RP is mainly due to a nutrient imbalance. Therapies aimed at increasing mTORC1 activity in cones or increasing the expression of key metabolic target genes of $\mathrm{mTORC} 1$ are thus plausible strategies to prolong vision in humans suffering from RP.

\section{Results}

$m$ TORC1 is required and sufficient to promote cone survival in RP. To evaluate whether insulin acts directly on cones through the insulin/mTOR pathway and to test whether continued stimulation of the pathway significantly prolongs cone survival, we constitutively activated the pathway in cones of $r d 1$ mice by conditional deletion of the tumor-suppressor gene phosphatase and tension homolog (Pten) using the Cre-lox system $(21,27)$ ( $r d 1$ M-opsin-Cre $\mathrm{Pten}^{\mathrm{f} / \mathrm{fl}}$ mice, herein referred to as $r d 1 \mathrm{Pten}^{c K O}$ mice. cKO denotes cone $\mathrm{KO}$; in all instances, $\mathrm{Cr}^{+}$denotes $\mathrm{cKO}$ of the gene indicated; the same cone-specific Cre driver line [M-opsin-Cre mice] [ref. 22] that was tested for efficient recombination [Supplemental Figure $1, \mathrm{~A}-\mathrm{E}]$ was used in all experiments that used conditional alleles). Pten is a phosphatase that counteracts the action of growth factors such as insulin by decreasing intracellular levels of the second messenger phosphatidylinositol-trisphosphate ( $\mathrm{PIP}_{3}$ ) (Figure 1). Therefore, activation of the pathway by loss of PTEN is not sensitive to the negative-feedback loop of the insulin/mTOR pathway, as $\mathrm{PIP}_{3}$ levels remain high even after growth factor receptors are turned off by the feedback loop (28). As a consequence of sustained pathway activity, we found that cone survival was significantly improved in retinae of $r d 1$ Pten $^{c K O}$ mice up to 8 months of age when compared with cone survival in $\mathrm{Cre}^{-}$littermate control retinae (Figure 2, A and B, and Supplemental Figure 2A) or with that observed in retinae following insulin injections in our previous study (17). The data suggest that sustained cell-autonomous activation of the pathway in cones can serve as a long-term therapeutic strategy to prolong cone survival in RP.

Loss of Pten activates various downstream signaling pathways and kinases. Two key kinases downstream of PTEN that are central to the insulin/mTOR pathway are protein kinase $\mathrm{B}$ (PKB), also known as AKT, and mTOR (21, 28-30). AKT has 3 isoforms that are often cell-type specific and a multitude of targets. In contrast, mTOR is found in 2 large protein complexes referred to as mTORC1 and mTORC2, each with a small, distinct set of known targets and characterized by their unique accessory proteins RAPTOR and RICTOR, respectively (21). Both AKT and mTORC2 respond mainly to growth factor changes and control many prosurvival mechanisms, while mTORC1 is growth factor and nutrient sensitive and regulates mainly cellular growth processes (21) (Figure 1). To identify which kinase(s) downstream of PTEN promote(s) cone survival, we first assessed the phosphorylation status of AKT and other bona fide targets of both mTOR complexes. Western blot analyses from whole retinal extracts at the onset of cone death (17) (P21) showed no appreciable phosphorylation changes, a result that could be explained by the low proportion of cones in the retina $(3 \%)(18,19,31)$ and/or the high expression levels of these phosphorylated proteins in other retinal cell types (Supplemental Figure 2, B and C). However, immunofluorescence on retinal flat mounts at P21 revealed an increase in the number of cones positive for 3-phosphosinositide-dependent protein kinase (PDK1) phosphorylation of AKT at Thr308 and a decrease in the number of cones positive for mTORC2-mediated phosphorylation of AKT at Ser473 (Figure 2C and ref. 21). Consistent with this finding, the number of cones positive for phosphorylated serum/glucocorticoid-regulated kinase 1 (p-SGK1), another mTORC2 target, was also reduced, while the number of cones phosphorylated on ribosomal protein S6 (p-S6), an indirect mTORC1 target (21), was increased (Figure 2C). Since phosphorylation of $\mathrm{AKT}$ on both sites is required for most AKT-mediated prosurvival functions, but phosphorylation on Thr308 is sufficient to activate mTORC1, the data suggest that cone survival upon loss of PTEN is mediated mainly by increased mTORC1 activity $(21,29,32)$. The reduction in mTORC2 activity upon loss of PTEN is likely due to the inhibitory effect of mTORC1 on mTORC2 $(33,34)$.

To test whether increased mTORC1 activity is responsible for the survival effect mediated by loss of PTEN, we generated rd1 mice with simultaneous deletion of Pten and Raptor in cones (rd1 M-opsin-Cre Pten ${ }^{f / f}$ Raptor ${ }^{f / f}$ allele; ref. 35 and Figure 1) and quantified cone survival in these mice at 2 months of age, a time point at which the difference in cone survival was quite large following loss of PTEN. Interestingly, concurrent loss of PTEN and RAPTOR not only abolished the survival effect seen upon loss of PTEN, but also accelerated cone death (Figure 3, A and B). A similar acceleration of cone death was seen upon loss of RAPTOR alone (Figure 3, A and B), while loss of RAPTOR in cones of WT mice did not affect cone survival at 2 months of age (data not shown). In contrast, loss of RICTOR (Rictor ${ }^{\text {flfl }}$ allele; ref. 36) in cones had no effect on cone survival in $r d 1$ (Figure 3, A and B) or WT mice (data not shown) at 2 months of age. Loss of RAPTOR and RICTOR was verified by phosphorylation changes in their downstream targets (21) S6, AKT, and SGK1 (Figure 3, C and D). The results show that mTORC1 activity is not only required for the improved cone survival seen upon loss of PTEN, but is also critical for cone survival under the stress conditions encountered during disease. Furthermore, the data suggest that 


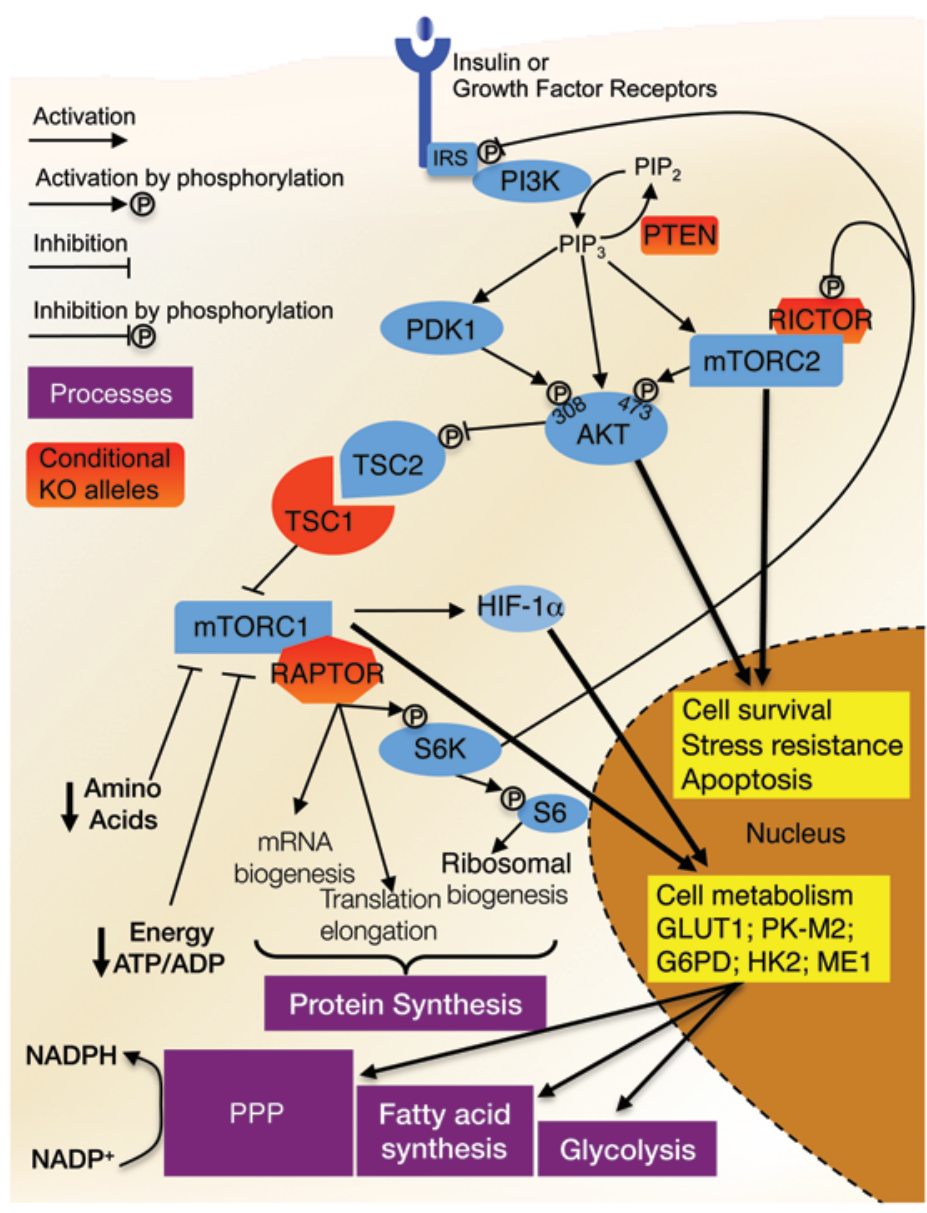

known mTORC2- and AKT-mediated prosurvival mechanisms do not contribute to cone survival upon loss of PTEN $(21,28,30)$.

To corroborate whether increasing mTORC1 activity alone is sufficient to prolong cone survival, we disrupted the tuberous sclerosis complex (TSC) (37), which is composed of the proteins TSC1 and TSC2 and is a negative regulator of mTORC1 activity (ref. 21 and Figure 1). Normally, the inhibitory effect of the complex on mTORC1 is released upon phosphorylation of TSC 2 by activated AKT (21). However, removal of either TSC1 or TSC2 alone results in a more robust and constitutive activation of mTORC1. Thus, we used mice with a conditional allele for Tsc1 to disrupt the TSC in cones of $r d 1$-mutant mice ( $r d 1 \mathrm{M}$-opsin-Cre $T s c 1^{f / / A}$ mice, herein referred to as $r d 1 \mathrm{Tsc}^{\mathrm{cKO}}$ ) (38). At 2 months of age, loss of TSC1 in these mice resulted in a more pronounced rescue of cones when compared with loss of PTEN (Figure 4, A and B). Many of the retinae displayed an almost WT distribution of cones in central areas. The protective effect was significant up to 8 months of age (Figure $4 \mathrm{~B})$. Cone function in these mice at 2 months of age, as evaluated by full-field electroretinography (ERG), was maintained at the levels recorded at the onset of cone death (P21), while it declined in $\mathrm{Cr}^{-}$animals (Figure 4C). ERG recordings were low in the $r d 1$ mutant at the onset of cone death when compared with those in WT mice ( $20 \%$ of WT), because rod degeneration starts before eye opening and before outer segments (OSs) have fully matured $(13,39,40)$. When compared with loss of PTEN, S6 was uniformly phosphorylated in cones, demonstrating a robust activation of
Figure 1. Schematic representation of the insulin/mTOR pathway. Upon insulin or growth factor binding, phosphosinositide 3-kinase $(\mathrm{PI} 3 \mathrm{~K})$ increases the concentration of the second messenger $\mathrm{PIP}_{3}$, a reaction that is reversed by PTEN. Increased PIP $_{3}$ levels promote mTORC2 and PDK1 activity. Both mTORC2 and PDK1 phosphorylate AKT on Ser473 and Thr308, respectively. While phosphorylation on both sites increases AKT activity, phosphorylation on Thr308 is sufficient to promote AKT activity toward TSC. Activated AKT phosphorylates the TSC protein tuberin (TSC2), which releases the inhibitory function of the TSC1/TSC2 complex on mTORC1, resulting in increased mTORC1 activity. Activated mTORC1 phosphorylates and thereby activates its target ribosomal protein S6 kinase (S6K). S6K negatively feeds back to growth factor receptors by phosphorylation of the insulin receptor substrate (IRS), which prevents overactivation of the pathway. Activated S6K can also phosphorylate RICTOR, which is part of the mTORC2 complex. Phosphorylation of RICTOR by S6K reduces mTORC2-mediated activation of AKT. mTORC1 controls mainly progrowth processes, while mTORC2 and AKT regulate many prosurvival and apoptotic processes.

mTORC1 in $r d 1$ Tsc ${ }^{c K O}$ retinae (Figure 4D). Loss of the protective effect by simultaneous ablation of TSC1 and RAPTOR, but not TSC1 and RICTOR, confirmed that mTORC1 activity was both required and sufficient to promote cone survival (Figure 5, A-C). Importantly, while mutations in TSC cause benign tumors in tissues such as the kidney and brain (37), loss of TSC1 in cones did not induce cone proliferation as assessed by the proliferation marker Ki67, indicating that the protection was due to improved cone survival and not cone numbers (Figure 5, D and E).

mTORC1 prolongs cone survival by improving cell metabolism. Photoreceptors are among the most metabolically active cells in the human body (41), since they need to replenish membranes and proteins lost due to the daily shedding of their OSs (42). Consequently, photoreceptors require large quantities of glucose to synthesize sufficient amounts of NADPH to keep up with daily membrane synthesis. mTORC1 is at the center of cell growth and proliferation, regulating genes involved in glycolysis, the pentose phosphate pathway (PPP), and de novo lipid synthesis (43). We therefore investigated whether sustained mTORC1 activity increases the expression of key metabolic target genes that increase NADPH production in cones, thereby improving survival and function. As such, we analyzed the expression of glucose transporter 1 (GLUT1), which increases glucose uptake, hexokinase 2 (HK2), which phosphorylates glucose more effectively, and glucose-6-phosphate dehydrogenase (G6PD), which shunts glucose into the PPP for NADPH synthesis (44). Immunofluorescence analysis revealed an increase in the expression of all 3 aforementioned genes in cones of 2-month-old $r d 1 \mathrm{Tsc}^{c \mathrm{KO}}$ mice (Figure 6A and Supplemental Figure 3A). We did not observe this increase in immunofluorescence upon the concurrent loss of TSC1 and RAPTOR (Figure 6A), indicating that the increase was mTORC1 dependent. Other glycolytic genes that contribute to an increase in NADPH production (45), such as pyruvate kinase muscle isoform 2 (PKM2) and malic enzyme 1 (ME1), were also revealed by immunofluorescence to be upregulated in cones of $r d 1 \mathrm{Tsc} \mathrm{I}^{\mathrm{cKO}}$ mice at 2 months of age, as was an increase in the transcription factor hypoxia-inducible factor $1 \alpha$ (HIF-1 $\alpha)$, which regulates 

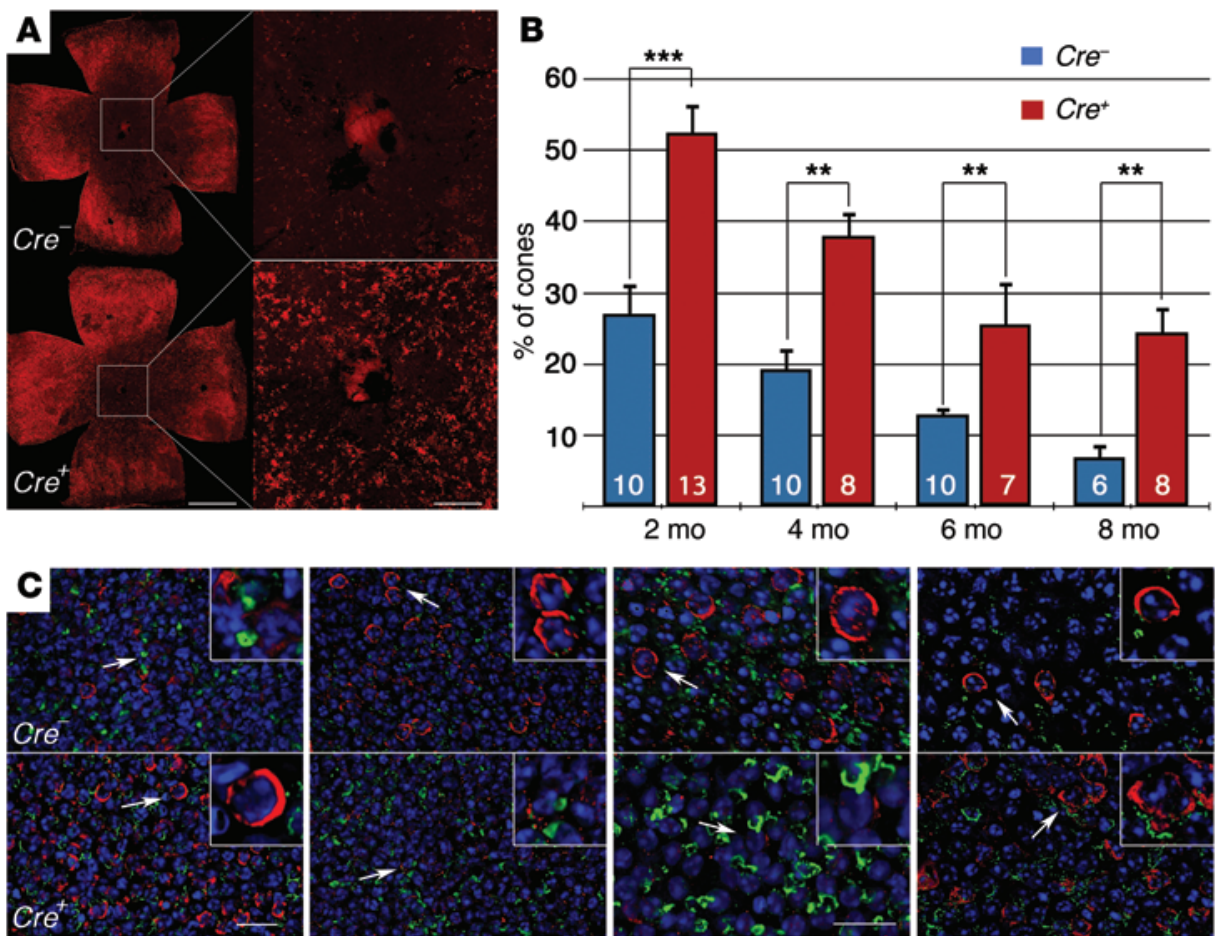

AKT (Thr308)

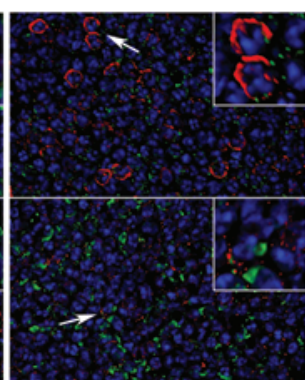

AKT (Ser473)

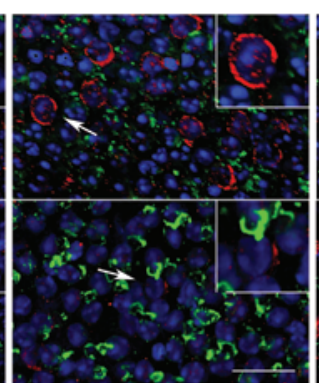

SGK (Ser422)

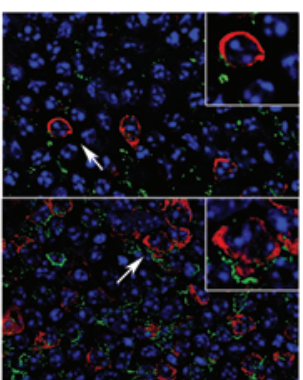

S6 (Ser240)
Figure 2. Loss of Pten promotes cone survival in rd1 mice. (A-C) rd1-mutant mice harboring the $\mathrm{Pten}^{\mathrm{fl} / \mathrm{fl}}$ allele. (A) Representative retinal flat mounts of $r d 1$-mutant mice at 2 months of age showing more central cones in $\mathrm{Cre}^{+}$animals (red signal indicates cone arrestin). Scale bar: $1 \mathrm{~mm}$ (left); $200 \mathrm{~mm}$ (right). (B) Quantification of cone survival at the indicated time points. Numbers in bars represent the number of retinae analyzed. ${ }^{* *} P<0.01$ and ${ }^{* * *} P<0.005$ by Student's $t$ test. (C) Immunofluorescence analyses at P21 of retinal flat mounts to detect phosphorylation of sites on the indicated proteins (red signal). Upper right of each panel shows magnification of the area indicated by an arrow. Cone layer is identified by PNA staining (green signal indicates PNA; blue signal indicates nuclear DAPI). Scale bars: $20 \mathrm{~mm}$ (original magnification for insets, $\times 2.5$ ). the transcription of many glycolytic genes (ref. 46, Supplemental Figure 3A, and Supplemental Figure 4). The data suggest that glucose uptake, retention, and divergence into the PPP are improved. In agreement with these findings, we detected significantly higher levels of NADPH in whole retinal extracts from $r d 1$ $T s c 1^{C K O}$ mice at P21 (Figure 6B), a time point at which cone death initiates but at which cone distribution was still similar between $\mathrm{Cre}^{-}$and $\mathrm{Cr}^{+}$littermate controls (Supplemental Figure 5). Interestingly, among the aforementioned metabolic genes, immunofluorescence showed that only ME1 expression was increased at P21 (Supplemental Figure 6A). This suggests that while mTORC1 signaling is active at P21 (Figure 4D), the expression of these metabolic genes increases gradually over time.

To further test this finding, we performed quantitative RT-PCR (qRT-PCR) analysis at P21 and P24 and found that most metabolic genes started to display a modest but statistically significant difference between $\mathrm{Cr}^{-}$and $\mathrm{Cr}^{+}$retinae by P24 (Supplemental Figure 6, B and C). Performing the qRT-PCR or NADPH assay at a later time point would have complicated the interpretation of the data, as the differences in cone density between $\mathrm{Cre}^{-}$ and $\mathrm{Cr}^{+}$retinae became statistically significant after P24 (Supplemental Figure 5), and thus any difference could be attributed to the greater number of cones. In agreement with an improvement in NADPH synthesis as well as a progressive increase in metabolic gene expression, we found that OS length was maintained in $\mathrm{Cre}^{+}$mouse retinae between P21 and 2 months of age (Figure 6, C and D). Since NADPH is also essential for chromophore recycling $(47,48)$, its increase, in conjunction with maintained OS length and a higher number of cones, may account for the higher ERG recordings in $\mathrm{Cre}^{+}$animals at 2 months of age. Interestingly, we did not detect any significant increase in
NADPH in retinal extracts from WT mice in which TSC1 was ablated in cones by using the same Cre driver line (Figure 6B). Consistent with this, loss of TSC1 did not cause an increase in the expression of any of these genes in the cones of WT mice by 2 months of age (Supplemental Figure 3B). However, phosphorylated S6 (p-S6) was readily detectable in the cones of WT mice lacking TSC1 (data not shown), confirming that mTORC1 activity was increased. In summary, sustained activation of mTORC1 in cones improves cone survival and function under disease conditions by gradually increasing the expression of genes involved in glucose uptake, retention, and utilization.

To further test whether NADPH levels are crucial for cone survival during disease, we examined the role of the initiator caspase, CASP2, a protease that initiates apoptotic cell death and has been shown to be activated under low intracellular NADPH levels but not low ATP levels $(24,25)$. Caspases are commonly activated by cleavage, thus the identification of a cleavage product is generally used to assess caspase activity (49). Western blot analysis using retinal extracts from $r d 1$ mice revealed no cleaved CASP2 products, while cleaved CASP2 was readily detectable after transfection of HEK239 cells with full-length Casp2 (Figure 7A), suggesting that CASP2 is not cleaved during cone degeneration. However, because dimerization of initiator caspases is sufficient to activate the protease, albeit to a lesser extent, the absence of a cleavage product does not necessarily mean that a caspase is not active (49). To further test whether CASP2 was activated in cones, we performed immunofluorescence analyses using 6 different CASP2 antibodies, none of which revealed any specific pattern of loss of CASP2 expression in the retinae of Casp $2^{-/-}$mice, making it difficult to determine whether CASP2 was actually expressed and activated in cones (data not 

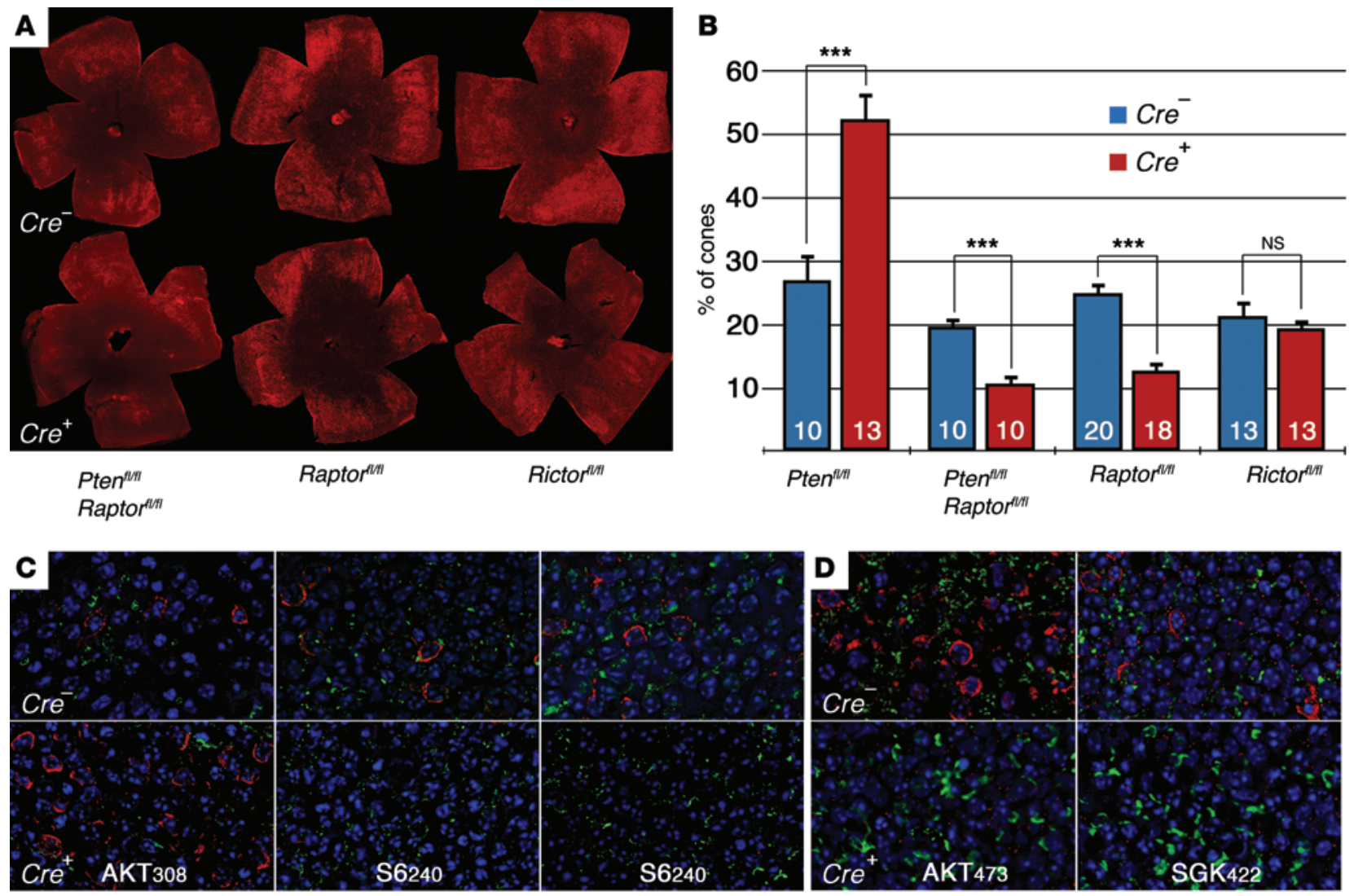

Pten $^{\text {AII }}$

Raptorim

Pten

Raptorim

Raptor ${ }^{\text {(I/W }}$

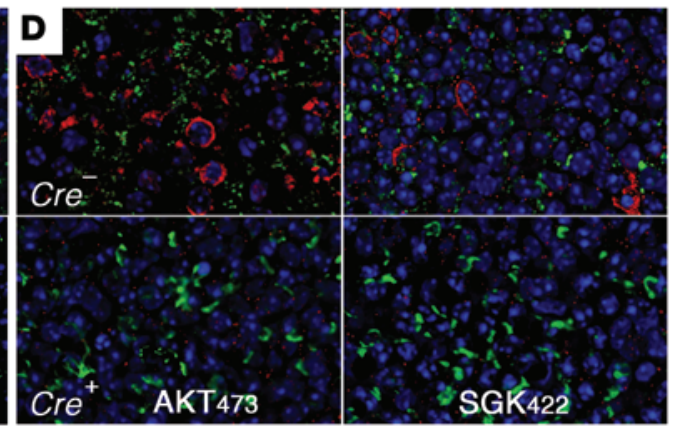

Rictor $^{\text {RII }}$

Figure 3. Raptor but not Rictor is required for loss of Pten-mediated survival. Data shown are from rd1-mutant mice harboring the indicated conditional alleles. (A and B) Representative retinal flat mounts (A) and quantification of cone survival (B) at 2 months of age (red signal indicates cone arrestin). Numbers in bars represent the number of retinae analyzed. ${ }^{* *} P<0.005$ by Student's $t$ test. (C) Immunofluorescence analyses (red signal) of retinal flat mounts at P21 to detect phospho-specific sites on the indicated proteins(green signal indicates PNA; blue signal indicates nuclear DAPI).

shown). However, Western blot analyses using extracts of the photoreceptor-enriched retinoblastoma cell line $\mathrm{Y} 79$ revealed an immunoreactive band (Figure 7A), suggesting that CASP2 may be present in photoreceptors. To determine whether CASP2 was activated in cones without cleavage, we used a peptide bearing the CASP2 target sequence (VDVAD) conjugated with the fluorophore FITC. The peptide becomes covalently linked once bound to the active site of the protease, facilitating the clearance of unbound excess peptide. Using this assay, we found that CASP2 activity occurred mainly in cones that lacked red-green opsin expression and displayed pyknotic nuclei, a key characteristic of apoptotic cells (Figure 7B). Moreover, the pattern of cells positive for CASP2 activity reflected the central-to-peripheral progression of cone death, suggesting that we were indeed capturing dying cones across the retina. However, because the sequence of caspase-binding peptides is rather short, false-positives can occur. To test for specificity of the activity assay and to determine whether Casp2 plays a role in vivo during cone degeneration, we crossed the Casp $2^{-/-}$allele onto an $r d 1$-mutant background (Casp2 $2^{-/} r d 1$ ). Loss of CASP2 not only abolished any CASP2 activity seen in $r d 1$ retinae (Figure 7B), but also significantly improved cone survival in the retinae of these mice at 20 weeks of age (Figure 7, C and D), while loss of CASP2 in WT mice did not affect photoreceptor survival (Figure 7E). In summary, the data suggest that removal of an NADPH-sensitive cell death mechanism can delay cone death in RP, further supporting the notion that cone death in RP is intimately linked to glucose and NADPH levels and indicating that this cone death is likely a result of nutrient deprivation.

Constitutively activated mTORC1 delays cone death in rhodopsin-KO mice. To test whether improved cone survival mediated by increased mTORC1 activity is applicable to other models of RP, we used the rhodopsin-KO (Rho-/) (23) mouse, which displays slower degeneration kinetics. Cone death initiates at around 17 weeks of age, and by 30 weeks of age, $\mathrm{Rho}^{-/-}$retinae show a degree of cone degeneration equivalent to that of 2-month-old $r d 1$ mouse retinae (17). Similar to the observations made in the $r d 1$ mouse model, loss of TSC1 in the cones of $\mathrm{Rho}^{-/-}$mice was able to significantly prolong cone survival at 30 weeks of age (Figure 8, A and B). Moreover, increased immunoreactivity against metabolic genes such as HK2 and PKM2 in cones, as well as an increase in the number of cones positive for p-S6 (Figure $8 \mathrm{C}$ ), indicates that the mechanism of protection is similar to that observed in $r d 1$ cones. These findings suggest that this approach is independent of the mutation in a rod-specific gene, allowing for therapeutic intervention at the mTORC1 level to prolong vision in RP. 

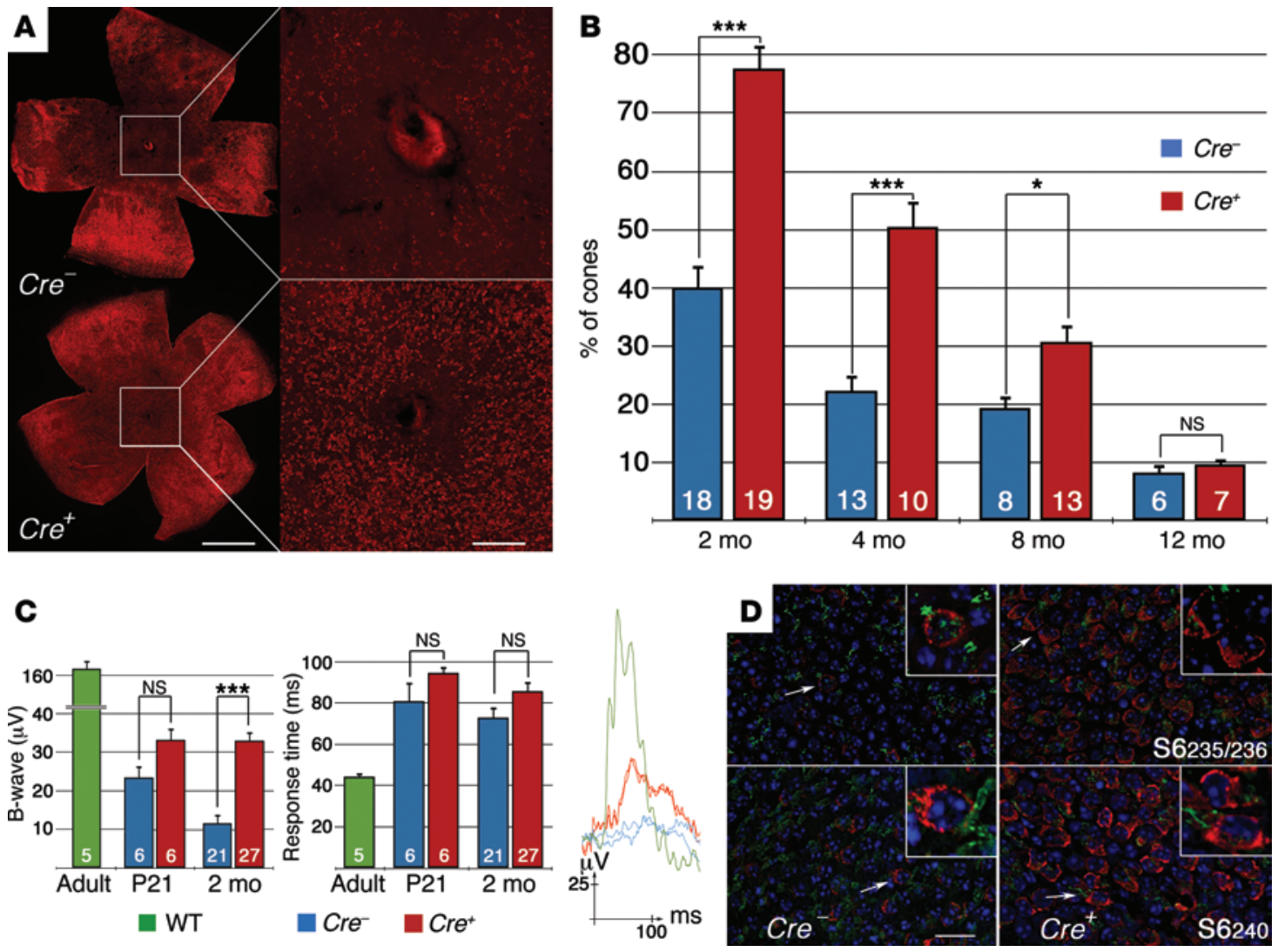

Figure 4. Activation of mTORC1 promotes cone survival and maintains cone function in rd1-mutant mice. (A-D) rd1-mutant mice harboring the $T_{s c 1^{f / f l}}$ allele. (A) Representative retinal flat mounts from rd1-mutant mice at 2 months of age showing a higher concentration of central cones in $\mathrm{Cre}^{+}$ animals (red signal indicates cone arrestin). Scale bars: $1 \mathrm{~mm}$ (left); $200 \mathrm{~mm}$ (right). (B) Quantification of cone survival at the indicated time points. Numbers in the bars represent the number of retinae analyzed. ${ }^{*} P<0.05$ and ${ }^{* *} P<0.005$ by Student's $t$ test. (C) Evaluation of cone function by photopic ERG recordings in mice at P21 and 2 months of age showing b-wave amplitude, the average response time of the b-wave peak, and representative recordings in animals at 2 months of age (left to right). The same ERG protocol was used for WT and $r d 1$ animals. Numbers in the bars represent the number of animals analyzed. ${ }^{* *} P<0.005$ by Student's $t$ test. (D) Immunofluorescence analyses to detect $p$-S6 on 2 different mTORC1-dependent sites (red signal) at P21 (green indicates PNA; blue indicates nuclear DAPI). Scale bar: $20 \mathrm{~mm}$ (original magnification for insets, $\times 2.5$ ).

\section{Discussion}

In this study, we show that cell-autonomous activation of metabolic genes downstream of mTORC1 is sufficient to prolong cone survival in RP, suggesting that improving nutrient uptake, retention, and utilization in cones delays cone death during disease. Our data showing an almost WT distribution of cones upon loss of TSC1 in mice at 2 months of age hold great promise, as the $r d 1$ mouse model is one of the fastest-progressing models of RP. Because cone death is also significantly delayed in the slow-progressing $\mathrm{Rho}^{-/-}$model of RP, the approach is predicted to be mutation independent and thus to improve cone survival across a wide spectrum of RP diseases in which the primary mutation is in a rod-specific gene.

Loss of the TSC has been shown in cell culture systems to render cells sensitive to complete glucose withdrawal, since mTORC1 fails to balance demand with supply (50). However, low nutrient conditions such as reduced glucose levels confer a survival advantage to cells that lose the TSC (51). This is because sustained mTORC1 activity under nutrient-restrictive conditions maintains protein synthesis and therefore allows for increased expression of transporters and other metabolic genes that help counterbalance the nutrient shortage. Similar to the findings in cell culture, loss of TSC in mouse cones confers a survival advantage during disease and increases the expression of transporters and metabolic genes. Therefore, increased mTORC1 activity helps starving cones to balance nutrient demand with supply. Consistent with this idea, loss of mTORC1 activity accelerates cone death in RP as cones fail to adapt to the nutrient shortage, while its activity is not required to keep cones alive in WT mice up to 2 months of age, since cones in these mice do not experience a nutrient shortage. Thus, the basal expression of many of these metabolic genes does not seem be to regulated by mTORC1 in cones, since its loss does not affect cones of WT mice up to 2 months of age. Our data suggest that the induction of mTORC1 target genes upon loss of TSC1 functions as an adaptive response in cones to counter nutrient stress. This idea is further supported by the finding that the expression of these metabolic genes increases gradually from P21 onward and is seen only in $r d 1$ and not WT retinae upon loss of TSC1. While this may appear at odds with in vitro data, in which loss of the TSC automatically increases the expression of mTORC1 targets (43), it is important to note that our experiments were carried out in vivo in postmitotic neurons within the context of a tissue. Thus cell 


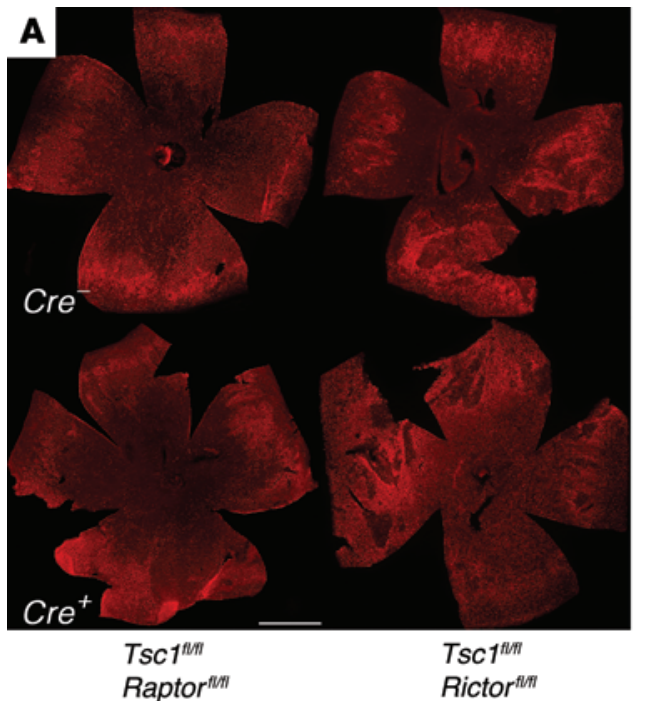

B
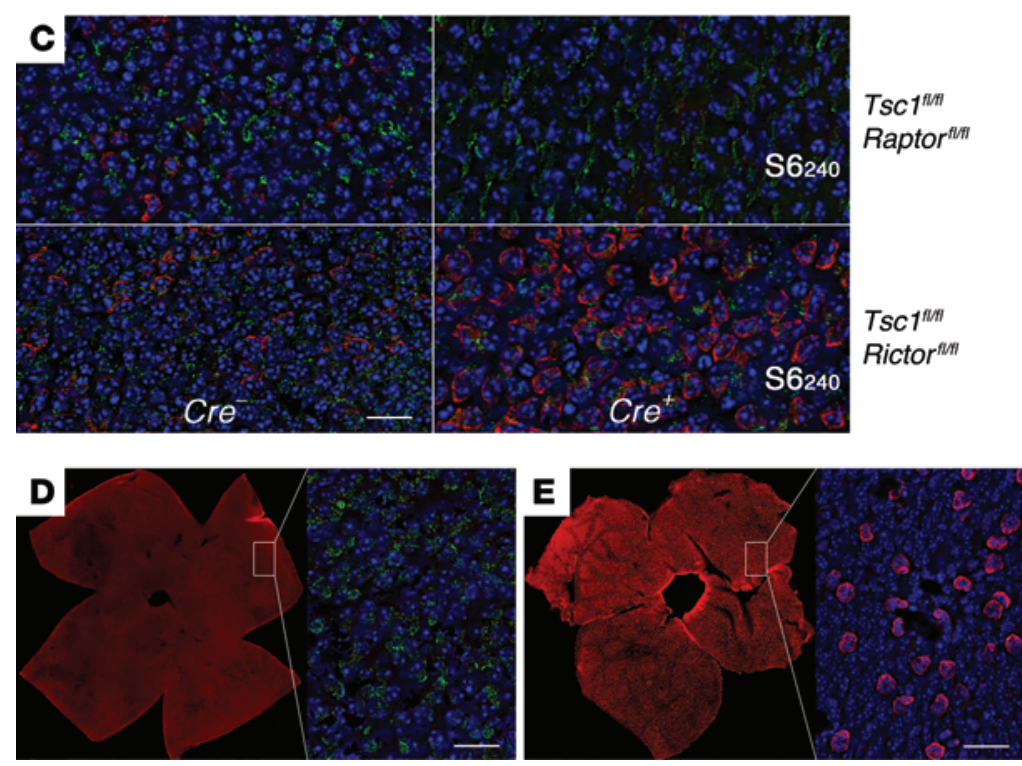

type-specific differences may account for this discrepancy. Similarly, loss of PTEN, which generally increases the activity of both mTOR complexes and AKT (21), results in decreased mTORC2 activity and decreased p-AKT (Ser473) in cones.

Cellular redox potential is tightly linked to nutrient availability. For example, NADPH is used to reduce oxidized glutathione, which serves to scavenge many free radicals. Increased NADPH levels upon loss of TSC1 likely contribute to cone survival through 2 different mechanisms. First, because NADPH is required for lipid and thus OS synthesis, increased levels can help prevent the OS shedding rates exceed the synthesis rate, thereby maintaining OS length, which can improve photoreceptor function. Photoreceptor function is also directly linked to NADPH, since NADPH is required to recycle the visual chromophore $(47,48)$. An overall healthier and better-functioning photoreceptor is likely to survive longer. Second, because oxidative stress is known to occur during the periods of cone degeneration, increased NADPH levels can directly reduce oxidative stress by increasing glutathione availability (52). Therefore, starvation of cones in RP is consistent with
Figure 5. mTORC1 is sufficient to prolong cone survival in RP. (A-C) Data shown are from mice on an rd1-mutant background harboring the indicated conditional alleles. (A) Representative retinal flat mounts from mice at 2 months of age (red signal indicates cone arrestin). Scale bar: $1 \mathrm{~mm}$. (B) Quantification of cone survival in mice at 2 months of age. Numbers in the bars represent number of retinae analyzed. ${ }^{*} P<0.05$ and ${ }^{* *} P<0.005$ by Student's $t$ test. (C) Immunofluorescence analyses of retinal flat mounts to detect $\mathrm{p}-\mathrm{S} 6$ at P21. p-S6 was absent from the cone layer (green signal indicates PNA) upon loss of TSC1 and RAPTOR, while the number of cones positive for $\mathrm{p}-\mathrm{S} 6$ increased upon loss of TSC1 and RICTOR (blue signal indicates nuclear DAPI). Scale bar: $20 \mathrm{~mm}$. ( $\mathbf{D}$ and $\mathbf{E}$ ) Ki67 staining (red signal) in retinae of $r d 1$ $T_{S C} 7^{\mathrm{CKO} O}$ mice at 2 months of age (D) and WT mice at PO (E), when cell division is ongoing (green signal indicates PNA; blue signal indicates nuclear DAPI). Higher magnification $(\times 30)$ is shown on the right side of each panel. Scale bar: $20 \mathrm{~mm}$.

a model of increased oxidative stress as a contributing factor to cone death (52). However, oxidative stress itself is unlikely to be the sole cause for cone death in $\mathrm{RP}$, since antioxidant treatments have had limited success in prolonging cone survival (13-16).

An understanding of the cell death mechanisms for cone death in RP remains largely elusive. Identifying a cell death mechanism may shed light on the overall cause of cell death, as some mechanisms require specific stress conditions or triggers. In this regard, the identification of Casp2 as the first apoptotic mechanism for cone death in RP further corroborates the notion that low NADPH levels are a contributing factor to cone death and supports the overall idea of a nutrient shortage in cones. However, a delay in cone death is only seen at later stages. This could be due in part to the involvement of necrosis during the initial phase of cone death. A recent report showed that early cone death in the $r d 10$ mouse model of RP includes necrotic cell death (53). The $r d 10$ model harbors a different mutation in the same gene as that in the $r d 1$ model; however, rod death progresses slightly slower than in the $r d 1$ model, yet is still faster than in the $R h^{-/-}$model. The electron microscopic analyses performed in that study revealed several cone nuclei with necrotic features at early stages of cone death. Additionally, the study showed that inhibition of necrosis reduces the number of necrotic nuclei and delays early cone death. Nonetheless, a greater number of cone nuclei showed signs of apoptotic cell death, even at early stages. Early necrotic cone death could be due to the rapidly occurring structural insult on the retina because of the rather rapid loss of rods. Once the necrotic phase ceases, the remaining cones may die by apoptotic mechanisms, including the activation of CASP2 induced by low NADPH levels. Early necrosis could explain why cone death progresses rapidly within the first days (P21-P28; Supplemental Figure 5), before activation of mTORC1 turns on the metabolic transcriptional network that delays cone death upon loss of TSC1. In combination with early necrosis, there are likely additional, as yet unidentified cell death mechanisms that contribute to cone death. These could explain why loss of CASP2 does not result in a 


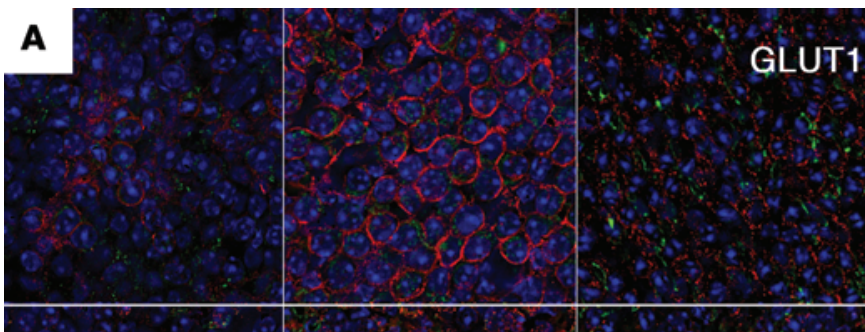

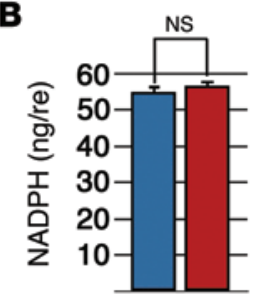

$T s c 1^{\text {Al }}$

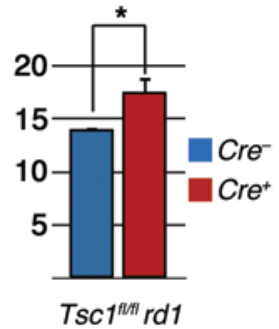

C

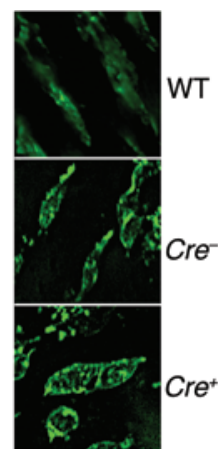

D
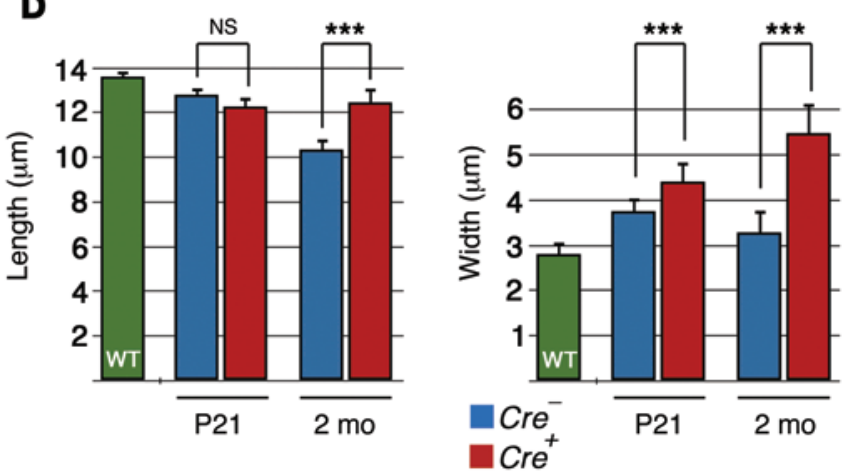

Figure 6. mTORC1 activation improves glucose metabolism in cones. (A) Immunofluorescence analyses to detect the indicated proteins (red signal) on retinal flat mounts from 2-month-old $r d 1$ mice harboring the indicated conditional alleles. Concurrent loss of TSC1 and RAPTOR abolished the increase in immunoreactivity seen upon loss of TSC1 alone (green signal indicates PNA; blue signal indicates nuclear DAPI). Scale bar: 20 mm. (B) NADPH measurements on whole retinal extracts at P21 from Tsc $7^{f / f l}$ mice on WT and $r d 1$-mutant backgrounds (ng/re, nanogram per retina). Error bars represent SD. ${ }^{*} P<0.05$ by Student's $t$ est. Data are representative of 3 biological replicas, with 3 retinae per replica. (C) Representative images of OSs from WT (top panel) and $r d 1$ Tsc $7^{f / f l}$ mice (middle and bottom panels) at 2 months of age. (D) Quantification of OS length and width at the indicated time points. Error bars represent SD. ${ }^{* *} P<0.005$ by Student's $t$ test. Data represent 40 measurements performed on 2 animals.

more profound effect. One would expect these mechanisms to be linked either directly or indirectly to low intracellular energy levels, as improving cell metabolism through activation of mTORC1 effectively delays cone death. For example, reduced NADPH levels could induce cell death through oxidative stress before CASP2 is activated. Thus, removal of one of the cell death mechanisms such as loss of CASP2, or treatments with antioxidants, is not sufficient to achieve the same effect that is seen with treating the overarching problem of starvation through loss of TSC1.

In summary, activation of the metabolic gene regulatory network downstream of mTORC1 significantly improved cone survival in a cell-autonomous manner, suggesting that the overall cause of cone death was nutrient shortage, in particular, a glucose shortage. Cone cell death itself is likely executed by a multitude of mechanisms, since in addition to the nutrient shortage, cones experience a variety of insults such as increased oxidative stress that occur at the same time due to the massive loss of rods. The insult causing the largest stress in any given cone may then determine the cell death mechanism used. This may explain why individual approaches have only limited success in delaying cone death in RP. Although our findings may not be directly translatable, since cell type-specific loss of the tumor-suppressor genes Pten or $T s c 1$ is not advisable in humans, they may redirect efforts aimed at developing therapies for patients suffering from RP. For example, adeno-associated virus-mediated gene transfer of mTORC1 targets in cones may provide a feasible approach for future treatments in humans. This approach rests on the identification of the correct combination of target genes required to prolong cone survival. Alternatively, drugs that increase mTORC1 activity could be delivered to the retina through slow-releasing eye implants.

It is of interest to note that the most common form of photoreceptor degeneration and thus the major cause for blindness in the industrialized world, age-related macular degeneration (AMD), may result in photoreceptor death due to the same proposed mechanism by which cones die in RP. In AMD, RPE cell function and health are affected, as the accumulation of drusen between the RPE and choriocapillary impinges on nutrient transfer from the bloodstream to RPE cells (54). Because RPE cells are the main source of nutrients for photoreceptors, a reduction in nutrient availability to RPE cells inevitably affects photoreceptors, too. Thus AMD and RP, although having different etiologies, may both result in blindness caused by starving cones. Therefore, improving photoreceptor metabolism by increasing mTORC1 activity may be applicable not only to RP, but also holds great promise for treating the most common form of retinal degeneration in the industrialized world.

\section{Methods}

Animals. Mice were maintained on a 12-hour light/12-hour dark cycle with unrestricted access to food and water. Lighting conditions were 
A
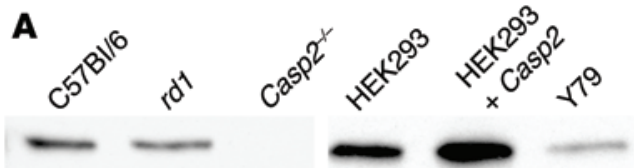

CAsF

CASP2

Cleaved

CASP2

Retinal extracts

Cell culture extracts

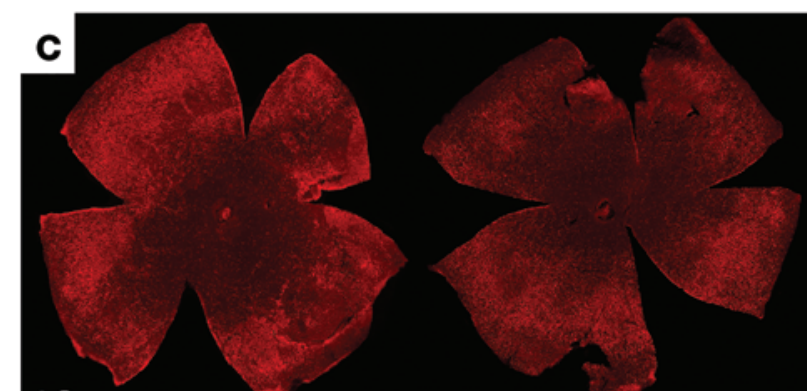

\section{$10 w$ \\ w}

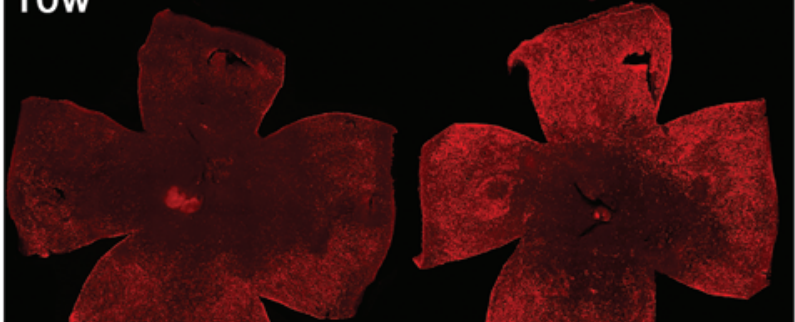

$20 w$

Casp2 $^{-1}$ rd1
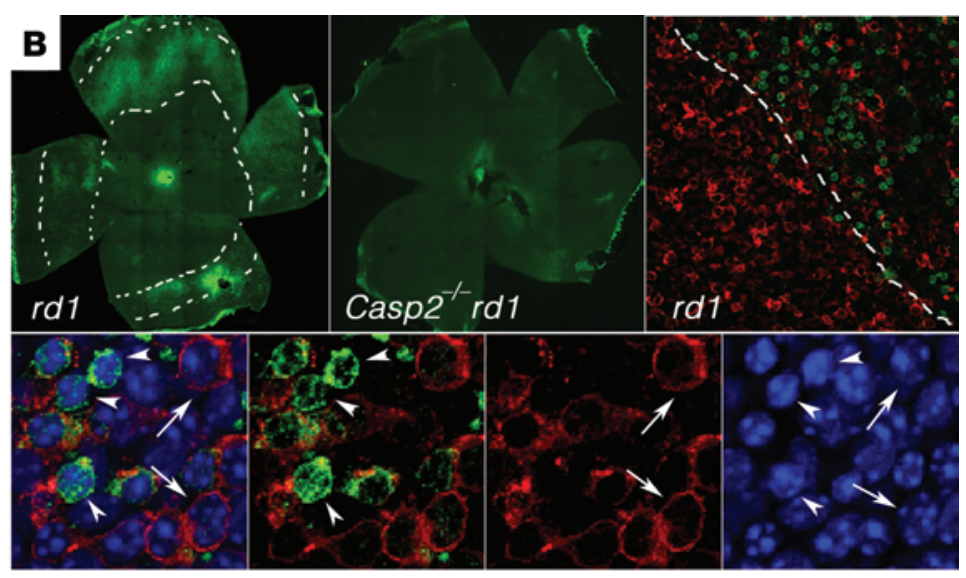

D

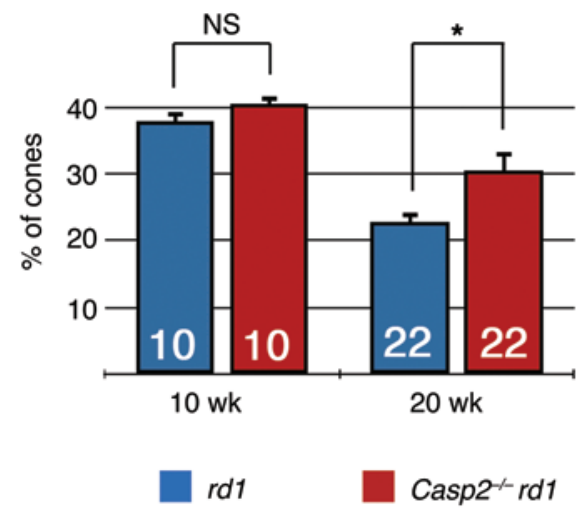

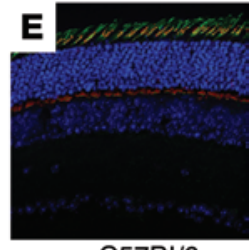

C57BI/6

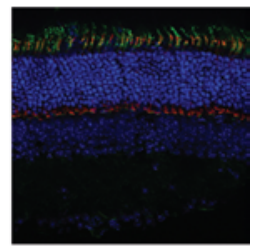

Casp2--

Figure 7. Loss of Casp2 slows cone death. (A) Western blot analyses of full-length and cleaved CASP2 in retinal extracts from the indicated genotypes (left blot) and in cell culture extracts (right blot) from HEK293 cells, HEK293 cells transfected with full-length Casp2, and in extracts from the photoreceptor-enriched retinoblastoma cell line Y79. (B) Immunofluorescence to detect active CASP2 (green signal indicates FITC-labeled CASP2 activity peptide) and red-green opsin (red signal) in retinae of 2-month-old animals. Top row, left to right: Retinal flat mount showing the CASP2-active zone (between the dotted lines) of cell death progressing toward the periphery. Middle panel: No CASP2 activity was detected in the absence of Casp2. Right panel: Higher magnification of central-to-peripheral death wave is demarked by the dotted line. To the left of the line, little CASP2 activity was seen, and many cells still expressed red-green opsin (red signal), while to the right of the line, there were few red-green opsin-positive cells and many cells positive for activated CASP2. Bottom panels: higher magnification showing that cells with more CASP2 activity (arrowheads) had pyknotic nuclei and less red-green opsin immunoreactivity as opposed to cells with more red-green opsin immunoreactivity (arrows; blue signal indicates nuclear DAPI). (C) Representative retinal flat mounts from mice of the indicated genotypes at 10 and 20 weeks of age. (D) Quantification of cone survival in rd1 and Casp2 ${ }^{-/-}$rd1 mice at the indicated time points. Numbers in the bars represent the number of retinae analyzed. ${ }^{*} P<0.05$ by Student's $t$ test. (E) Immunofluorescence analyses to detect cone arrestin (red signal) and PNA (green signal; blue signal indicates nuclear DAPI) in retinal cryosections from 20-week-old WT and Casp2 ${ }^{-/-}$mice.

kept constant in all cages, with illumination ranging between 10 and 15 lux. The Pde6b $b^{r d 1 / r d 1}$ ( $\left.r d 1\right)$, Casp2 $2^{-/}$, C57BL/6J, Pten ${ }^{f / f}, T s c 1^{f / f}$, and Ai9 Cre reporter mice were purchased from The Jackson Laboratory (20, 26, 27, 38, 55). The M-opsin-Cre (cone-specific Cre line using the human medium wavelength promoter), Raptor ${ }^{A / f}$, Rictor ${ }^{f / f}$, and $R h o^{-/-}$mice have been described previously by Yun Z. Le, Michael N. Hall, Markus A. Rüegg, and Janis Lem, respectively $(22,23,35)$. Genotyping was performed as described in the original publications. None of the mice used for analysis were on an albino background, and all mice were genotyped for absence of the $r d 8$ mutation (56). To dissect the role of the insulin/mTOR pathway in cone survival, the conditional allele was first crossed with the cone-specific Cre-driver line and then with the $r d 1$ line to generate $\mathrm{Cre}^{-}$and $\mathrm{Cre}^{+} \mathrm{rd1}$-conditional allele $(c K O)$ lines. In some cases, double-conditional alleles were also generated. The $\mathrm{Cr}^{+}$and $\mathrm{Cre}^{-}$lines were crossed with each other (e.g., rd1 Pten ${ }^{f / f} r d 1$ Pten $^{f / f l} \mathrm{M}$-opsin-Cre ${ }^{+/-}$Pten $^{f / f f}$ ) to generate the $\mathrm{Cre}^{+}$and $\mathrm{Cre}^{-}$littermates that were used for analyses. Because various strains were interbred, strain background differences may account for the slight variations in cone survival in $\mathrm{Cre}^{-}$animals at 2 months of age. Nonetheless, our data clearly show that mTORC1 was required for cone survival during disease, since loss of RAPTOR always accelerated cone death, while increasing mTORC1 activity consistently improved cone survival. Because loss of Casp2 did not allow us to compare $\mathrm{Cr}^{-}$and $\mathrm{Cr}^{+}$littermates, we generated a congenic $r d 1$ Casp $2^{-/-}$line to study the effect of loss of CASP2 on cone survival. The Casp $2^{-1-}$ allele was on a C57BL/6J background and was crossed with $r d 1 \mathrm{C} 57 \mathrm{BL} / 6 \mathrm{~J}$ mice that were generated by backcrossing the $P d e 6 b^{r d 1 / r d 1}$ allele of the FVB strain with C57BL/6J mice for 10 generations. $r d 1 \mathrm{C} 57 \mathrm{BL} / 6 \mathrm{~J}$ mice were then compared with $r d 1$ Casp $2^{-/-}$ C57BL/6J mice to quantify cone survival.

ERG. ERG was performed using the Espion E3 console in conjunction with the ColorDome (Diagnosys LLC). Mice were anesthetized 

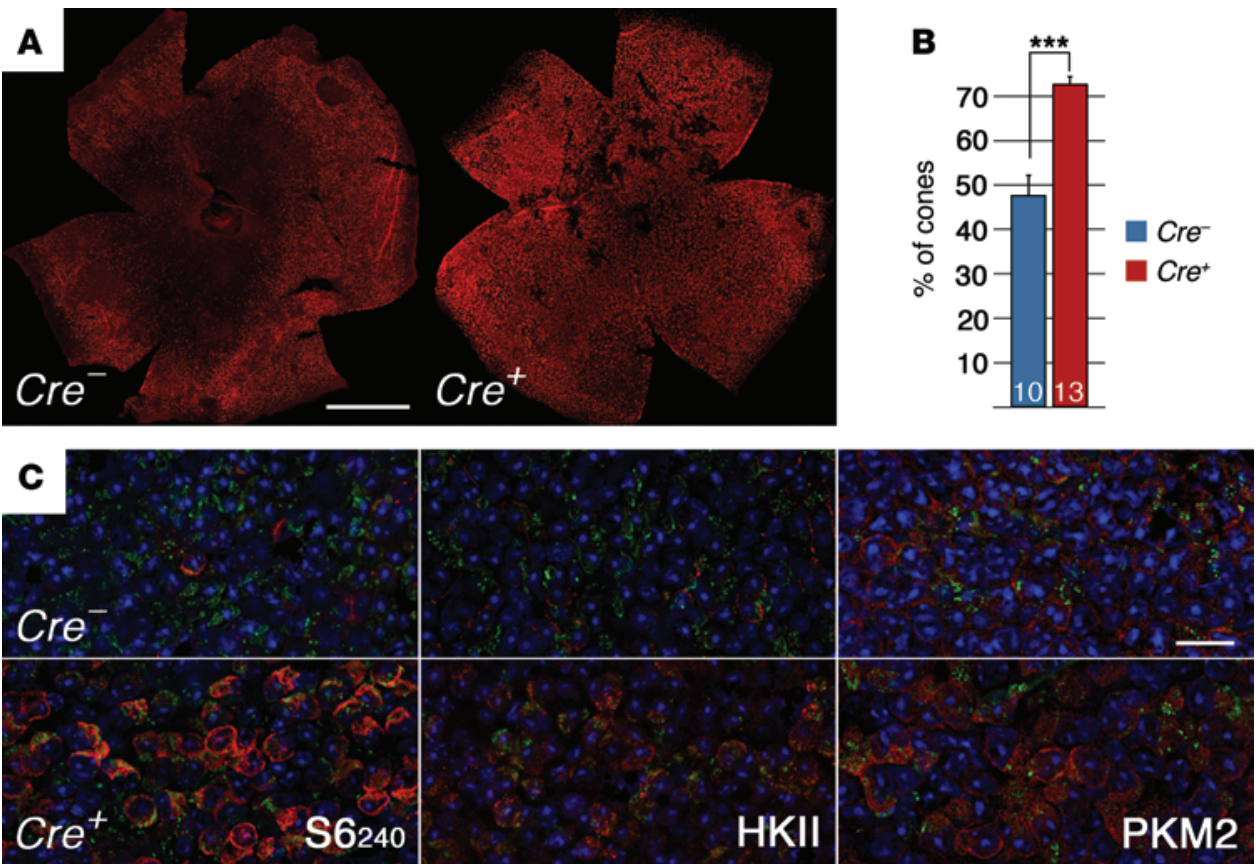

Figure 8. Cone protection mediated by loss of TSC1 is conserved in RP. Data shown are from $\mathrm{RhO}^{-/-}$mice harboring the $T s f^{f / / f l}$ allele. (A) Representative retinal flat mounts from mice at 30 weeks of age (red signal indicates cone arrestin). Scale bar: $1 \mathrm{~mm}$. (B) Quantification of cone survival in mice at 30 weeks of age. Numbers in the bars represent the number of retinae analyzed. ${ }^{* * *} P<0.005$ by Student's $t$ test. (C) Immunofluorescence analyses to detect the indicated proteins (red signal) on retinal flat mounts (green signal indicates PNA; blue signal indicates nuclear DAPI). Scale bar: $20 \mathrm{~mm}$.

lectin (PNA) (1:500; catalog FL-1071; Vector Laboratories). All secondary antibodies (donkey) were purchased from Jackson ImmunoResearch and were purified $\mathrm{F}(\mathrm{ab})_{2}$ fragments that displayed minimal cross-reactivity with other species.

Quantification of cone survival. The

by an i.p. injection of a ketamine-xylazine $(100 \mathrm{mg} / \mathrm{kg}$ and $10 \mathrm{mg} / \mathrm{kg}$, respectively) mixture. One drop each of phenylephrine $(2.5 \%)$ and tropicamide (1\%) was applied for pupil dilation 10 minutes before to recording. Animals were kept on a warming plate during the entire ERG procedure to maintain body temperature at $37^{\circ} \mathrm{C}$. Photopic ERGs were recorded after light adaptation with a background illumination of $34 \mathrm{~cd} / \mathrm{m}^{2}$ (white $6500 \mathrm{~K}$, produced by a Ganzfeld stimulator) for 8 minutes. Flashes were presented at 1-minute intervals with a pulse length of $4 \mathrm{~ms}$, and each recording consisted of a single flash of $35 \mathrm{~cd} \times \mathrm{s} / \mathrm{m}^{2}$. Six to nine trials were averaged for single-flash responses.

Histological methods. Antibody stainings on retinal cryosections and retinal flat mounts were performed as described previously (17, 57). CASP2 activity on retinal whole mounts was detected using the Green FLICA Caspase 2 Activity Kit (catalog 918; ImmunoChemistry Technologies) according to the manufacturer's instructions. Briefly, retinae were incubated in DMEM media with the detection reagent at $37^{\circ} \mathrm{C}$ for 30 minutes, followed by 2 washes of 5 minutes each in apoptosis wash buffer. Retinae were then fixed and processed for antibody staining as described $(17,57)$. Antibody stainings were performed in TBS buffer for phospho-specific antibodies and in PBS buffer for all other antibodies. The following primary antibodies and concentrations were used: rabbit $\alpha-$-AKT (Thr308) (1:1,000; cata$\log 2965)$; rabbit $\alpha$-p-AKT (Ser473) (1:1,000; catalog 4060); rabbit $\alpha$-p-S6 (Ser240) (1:300; catalog 5364); rabbit $\alpha$-p-S6 (Ser235/236) (1:300; catalog 4856); rabbit $\alpha$-PKM2 (1:300; catalog 4053); rabbit $\alpha$-HK2 (1:300; catalog 2867) (all from Cell Signaling Technology); rabbit $\alpha$-p-SGK (Ser422) (1:300; catalog ab55281); rabbit $\alpha$-G6PD (1:300; catalog ab993); rabbit $\alpha$-ME1 (1:300; catalog ab97445); rabbit $\alpha$-Ki67 (1:300; catalog ab15580) (all from Abcam); rabbit $\alpha$-HIF-1 $\alpha$ (1:300; catalog ab1536; R\&D Systems); rabbit $\alpha$-GLUT1 (1:30; catalog GT11-A; Alpha Diagnostics); mouse $\alpha$-Cre (1:500; catalog MMS-106P; Covance); rabbit $\alpha$-cone arrestin (1:300; catalog AB15282; EMD Millipore); rabbit $\alpha$-red-green opsin (1:300; catalog AB5405; EMD Millipore); and fluorescein-labeled peanut agglutinin
Ai9 Cre-reporter line was used to validate uniform expression of Cre recombinase across the retina (Supplemental Figure 1, A-E, and ref. 55). Quantification of cone survival by colocalization was performed using a method similar to one described previously (17). In brief, the percentage of surviving cones is based on a calculation of the percentage of the retinal surface area that is covered by cones. To determine the area that was covered by cones, we used the staining detected by the cone arrestin antibody (Supplemental Figure 1, F and G: red signal) as a proxy for surviving cones. To determine the retinal surface area, retinae were stained with PNA-coupled FITC (Supplemental Figure 1, F and G: green signal in the first and last columns and gray scale signal in the third column) at a dilution that was 3 times higher than that used for all other staining procedures, with the goal to only elicit sufficient background fluorescence to highlight the entire retinal surface area rather than detect cone OSs. The ratio of red to green pixels, which was calculated using CoLocalizer Pro software (CoLocalization Research Software) (58) with its integrated background correction, thus directly representing the percentage of the retinal surface area that was covered by the cone arrestin antibody staining. Before calculating the percentage of colocalization of red and green, the upper and lower thresholds of each signal were adjusted, such that only cones were visible in the red signal (Supplemental Figure 1, $\mathrm{H}$ and I), and the entire retina was visible in the green signal. To calibrate the percentage of colocalization of red and green signals on an $r d 1$ mutant background to the actual percentage of surviving cones, we performed a real cone count rather than determining the percentage of cone arrestin staining that covered the retina in WT mice. The reason for this is that during degeneration, cone arrestin was also found in the cell body, resulting in a different pattern of expression; thus, the percentage of colocalization was different when compared with that in retinae of WT mice (Supplemental Figure 1, J and K). A cone count was performed across $3 \mathrm{WT}$ retinae and across $3 \mathrm{rd1} \mathrm{Tsc}^{1 \text { cKO }}$ retinae at P21 (Supplemental Figure 1, L-N). The mutant strain and time point were chosen at P21, since at this point, cone death had just started, 
and the percentage of colocalization of red and green signals was the highest upon loss of TSC1 (52.7\%). The actual cone count in WT retinae was in agreement with published data (59) and revealed that at $\mathrm{P} 21,95.2 \%$ of cones were still present in the $r d 1 \mathrm{Tsc} \mathrm{c}^{\mathrm{cKO}}$ retinae when compared with those in WT retinae, meaning that $52.7 \%$ of colocalization (red to green signal) corresponds to $95.2 \%$ of cones. These numbers were used to calibrate all colocalization data representing cone survival. To validate the method, we counted cones in retinae of $r d 1 T s c 1^{c K O}$ mice at 2 months of age (Supplemental Figure 1, L-N). This time point was chosen because upon loss of TSC1, cone survival at 2 months of age is still quite homogenous, rather than demonstrating a patchy pattern of survival, thus making it more accurate to determine the number of cones across the entire retina by counting individual fields. The colocalization and actual cone count data for mice at 2 months of age were in agreement, with no statistically significant difference between them $(77.6 \% \pm 3.9 \%$ vs. $75.8 \% \pm 3.7 \%$, respectively), indicating that the colocalization method can be used to determine the percentage of surviving cones. The method of quantifying cones by colocalization over the entire retinal surface, rather than counting the number of cones in a few individual fields, was chosen because cone degeneration is quite patchy, progressing from the center to the periphery. Therefore, counting a few individual fields, even if always done in the exact same location, is not likely to properly represent the number of surviving cones. Thus, this method is independent of the degeneration pattern and the orientation of the retina.

For quantification of cones by cone count, retinae were first divided into 5 different sectors with increasing radiuses of $500 \mathrm{~mm}$ per sector (Supplemental Figure 1L). Cones were then counted manually in 4 squares per sector (20 squares/retina), each square measuring $40,000 \mathrm{~mm}^{2}$, to determine the average cone density per sector $\left(\right.$ cones $\left./ \mathrm{mm}^{2}\right)$. The average cone density per sector was then multiplied with the sector surface area to obtain the number of cones per sector. The surface area of each sector was calculated as the sector surface area of a sphere. The total number of cones per retina was then obtained by adding the number of cones across the 5 sectors. The numbers shown represent an average of 3 retinae per genotype and age. Retinal flat-mount images for the colocalization analyses and cone count were acquired by tiling individual images taken at $\times 16$ magnification (Leica DM5500) over the entire retinal surface area with an automated scanning stage.

Western blot analysis. Retinae from 2 animals were pooled and homogenized by sonication in ristocetin-induced platelet aggregation (RIPA) buffer containing protease and phosphatase inhibitors (cOmplete Protease Inhibitor Cocktail and PhosSTOP Phosphatase Inhibitor Cocktail; both from Roche Diagnostics). Protein concentration was assessed using a Protein Assay Kit (Bio-Rad), and $10 \mu \mathrm{g}$ protein was loaded per lane. Proteins were separated on a 4\%-20\% Tris-Glycine gradient gel (Bio-Rad) at $170 \mathrm{~V}$ and transferred onto a nitrocellulose membrane for 2 hours at $4^{\circ} \mathrm{C}$ with a current of $150 \mathrm{~mA}$. Membrane was blocked for 1 hour at room temperature in $5 \%$ fat-free dry milk powder, incubated with primary antibody overnight at $4^{\circ} \mathrm{C}$, washed 3 times for 20 minutes each wash at room temperature, incubated with an HRP-coupled secondary antibody (1:10,000; Santa Cruz Biotechnology Inc.) for 2 hours, and washed 3 times for 20 minutes each wash at room temperature. Signal was detected with SuperSignal West Dura (Pierce Biotechnology). All incubations were performed in the presence of $0.1 \%$ Tween-20 and $5 \%$ fat-free dry milk powder. Incuba- tions were performed either in TBS buffer for phospho-specific antibodies or in PBS buffer for all other antibodies. The following primary antibodies were used: rabbit $\alpha$-p-AKT(308) (1:1,000; catalog 2965); rabbit $\alpha$-p-AKT(473) (1:1,000; catalog 4060); mouse $\alpha$-AKT (1:1,000; catalog 2967); rabbit $\alpha$-p-S6 (1:1,000; catalog 5364); and rabbit $\alpha$-S6 (1:1,000; catalog 2217) (all from Cell Signaling Technology); mouse $\alpha$ - $\beta$-actin (1:2,000; catalog A5316; Sigma-Aldrich); mouse $\alpha$-CRE (1:2,000; catalog 69050-3; Novagen); rabbit $\alpha$-cone arrestin (1:1,000,; catalog AB15282; EMD Millipore); and rat $\alpha$-CASP2 (1:1,000; catalog ALX-804-356; clone 11B4; Enzo Life Sciences).

qRT-PCR. RNA was isolated using TRIzol (Life Technologies) as described previously (17). Three biological samples of each genotype were analyzed, and each sample consisted of retinae from 2 different animals. cDNAs were generated using the Transcriptor First Strand cDNA Synthesis Kit (Roche Diagnostics), and SYBR green-based qRT-PCR (Kapa Biosystems) was performed using the Bio-Rad CFX96 Real-Time System. Samples were run in duplicate and normalized to $\beta$-actin using the $2^{-\Delta \Delta \mathrm{Ct}}$ method. The primer sets used are provided in Supplemental Table 1.

NADPH measurement. NADPH was measured using the Fluoro NADP/NADPH kit from Cell Technology Inc. (NADPH100-2). The assay was performed in triplicate using 3 biological samples, with each biological sample consisting of 3 retinae. Retinae were dissected in DMEM media, rinsed with $1 \mathrm{X}$ PBS, and processed following the manufacturer's instructions. Retinae were sonicated after the addition of the lysis buffer to ensure efficient dissociation.

Cell culture. Casp2 was amplified by PCR (forward: 5'-ATGGCGGCGCCGAGCGGGAGGTCG-3'; reverse: 5'-TCACGTGGGTGGGTAGCCTGGG-3') from a C57BL/6J retinal cDNA library that was generated as described previously (17). The PCR product (1359 bp) was subcloned into the pGEM-T easy vector (Promega) and sequence verified. The Casp2 cDNA was then cloned into an rAAV2 plasmid carrying a $C M V$ promoter and the SV40 polyadenylation site. HEK293 cells were transfected with PEI (23966; Polyscience Inc.) and maintained in regular DMEM media containing 10\% FCS and a penicillin-streptomycin mixture. The same media were used to maintain the Y79 cell line. Protein extractions were performed as described above.

Statistics. The Student's $t$ test was used for statistical analyses. $P$ values of less than 0.05 were considered statistically significant. If not otherwise noted in the figure legend, all error bars represent the SEM.

Study approval. The IACUC of the University of Massachusetts Medical School approved the animal study. All procedures involving animals were performed in compliance with the Association for Research in Vision and Ophthalmology (ARVO) Statement for the Use of Animals in Ophthalmic and Vision Research.

\section{Acknowledgments}

We would like to thank Eric Baehrecke, Francis Chan, Hemant Khanna, Daryl Bosco, David Guertin, Lolita Petit, and Marina Zieger for discussions, critical reading of the manuscript, and/or reagents. This work was supported by the NIH (RO1 EY023570).

Address correspondence to: Claudio Punzo, Department of Ophthalmology and Gene Therapy Center, University of Massachusetts Medical School, 368 Plantation Street, Worcester Massachusetts 01605, USA. Phone: 508.856.8038; E-mail: Claudio.Punzo@umassmed.edu. 
1. Biel M, et al. Selective loss of cone function in mice lacking the cyclic nucleotide-gated channel CNG3. Proc Natl Acad Sci U S A. 1999;96(13):7553-7557.

2. Yang RB, Robinson SW, Xiong WH, Yau KW, Birch DG, Garbers DL. Disruption of a retinal guanylyl cyclase gene leads to cone-specific dystrophy and paradoxical rod behavior. J Neurosci. 1999;19(14):5889-5897.

3. Cho KI, et al. Distinct and atypical intrinsic and extrinsic cell death pathways between photoreceptor cell types upon specific ablation of Ranbp 2 in cone photoreceptors. PLoS Genet. 2013;9(6):e1003555

4. Xu J, et al. CNGA3 deficiency affects cone synaptic terminal structure and function and leads to secondary rod dysfunction and degeneration. Invest Ophthalmol Vis Sci. 2012;53(3):1117-1129.

5. Hartong DT, Berson EL, Dryja TP. Retinitis pigmentosa. Lancet. 2006;368(9549):1795-1809.

6. Steinberg RH. Survival factors in retinal degenerations. Curr Opin Neurobiol. 1994;4(4):515-524.

7. Mohand-Said S, et al. Photoreceptor transplants increase host cone survival in the retinal degeneration (rd) mouse. Ophthalmic Res. 1997;29(5):290-297.

8. Mohand-Said S, et al. Normal retina releases a diffusible factor stimulating cone survival in the retinal degeneration mouse. Proc Natl Acad Sci U S A. 1998;95(14):8357-8362.

9. Mohand-Said S, Hicks D, Dreyfus H, Sahel JA. Selective transplantation of rods delays cone loss in a retinitis pigmentosa model. Arch Ophthalmol. 2000;118(6):807-811.

10. Leveillard $\mathrm{T}$, et al. Identification and characterization of rod-derived cone viability factor. Nat Genet 2004;36(7):755-759.

11. Streichert LC, Birnbach CD, Reh TA. A diffusible factor from normal retinal cells promotes rod photoreceptor survival in an in vitro model of retinitis pigmentosa. J Neurobiol. 1999;39(4):475-490.

12. Gupta N, Brown KE, Milam AH. Activated microglia in human retinitis pigmentosa, late-onset retinal degeneration, and age-related macular degeneration. Exp Eye Res. 2003;76(4):463-471.

13. Komeima K, Rogers BS, Lu L, Campochiaro PA. Antioxidants reduce cone cell death in a model of retinitis pigmentosa. Proc Natl Acad Sci U S A 2006;103(30):11300-11305.

14. Komeima K, Rogers BS, Campochiaro PA. Antioxidants slow photoreceptor cell death in mouse models of retinitis pigmentosa. J Cell Physiol. 2007;213(3):809-815.

15. $\mathrm{Lu} \mathrm{L}$, et al. Increased expression of glutathione peroxidase 4 strongly protects retina from oxidative damage. Antioxid Redox Signal. 2009;11(4):715-724.

16. Usui S, et al. Increased expression of catalase and superoxide dismutase 2 reduces cone cell death in retinitis pigmentosa. Mol Ther. 2009;17(5):778-786.

17. Punzo C, Kornacker K, Cepko CL. Stimulation of the insulin/mTOR pathway delays cone death in a mouse model of retinitis pigmentosa. Nat Neurosci. 2009;12(1):44-52.

18. Jeon CJ, Strettoi E, Masland RH. The major cell populations of the mouse retina. J Neurosci. 1998;18(21):8936-8946
19. Masland RH. The fundamental plan of the retina. Nat Neurosci. 2001;4(9):877-886.

20. Bowes C, Li T, Danciger M, Baxter LC, Applebury ML, Farber DB. Retinal degeneration in the rd mouse is caused by a defect in the beta subunit of rod cGMP-phosphodiesterase. Nature. 1990;347(6294):677-680.

21. Zoncu R, Efeyan A, Sabatini DM. mTOR: from growth signal integration to cancer, diabetes and ageing. Nat Rev Mol Cell Biol. 2011;12(1):21-35.

22. Le YZ, Ash JD, Al-Ubaidi MR, Chen Y, Ma JX, Anderson RE. Targeted expression of Cre recombinase to cone photoreceptors in transgenic mice. Mol Vis. 2004;10:1011-1018.

23. Lem J, et al. Morphological, physiological, and biochemical changes in rhodopsin knockout mice. Proc Natl Acad Sci U S A.1999; 96(2):736-741.

24. Nutt LK, et al. Metabolic regulation of oocyte cell death through the CaMKII-mediated phosphorylation of caspase-2. Cell. 2005; 123(1):89-103.

25. Nutt LK, et al. Metabolic control of oocyte apoptosis mediated by 14-3-3zeta-regulated dephosphorylation of caspase-2. Dev Cell. 2009;16(6):856-866.

26. Bergeron L, et al. Defects in regulation of apoptosis in caspase-2-deficient mice. Genes Dev. 1998;12(9):1304-1314.

27. Lesche R, et al. Cre/loxP-mediated inactivation of the murine Pten tumor suppressor gene. Genesis. 2002;32(2):148-149.

28. Hollander MC, Blumenthal GM, Dennis PA. PTEN loss in the continuum of common cancers, rare syndromes and mouse models. Nat Rev Cancer. 2011;11(4):289-301.

29. Hung CM, Garcia-Haro L, Sparks CA, Guertin DA. mTOR-dependent cell survival mechanisms. Cold Spring Harb Perspect Biol. 2012;4(12):a008771

30. Hers I, Vincent EE, Tavare JM. Akt signalling in health and disease. Cell Signal. 2011;23(10):1515-1527.

31. Masland RH. Cell populations of the retina: the Proctor lecture. Invest Ophthalmol Vis Sci. 2011;52(7):4581-4591.

32. Guertin DA, et al. Ablation in mice of the mTORC components raptor, rictor, or mLST8 reveals that $\mathrm{mTORC} 2$ is required for signaling to Akt-FOXO and PKC $\alpha$, but not S6K1. Dev Cell. 2006;11(6):859-871.

33. Dibble CC, Asara JM, Manning BD. Characterization of Rictor phosphorylation sites reveals direct regulation of mTOR complex 2 by S6K1. Mol Cell Biol. 2009;29(21):5657-5670.

34. Julien LA, Carriere A, Moreau J, Roux PP. mTORC1-activated S6K1 phosphorylates Rictor on threonine 1135 and regulates mTORC2 signaling. Mol Cell Biol. 2010;30(4):908-921.

35. Bentzinger CF, et al. Skeletal muscle-specific ablation of raptor, but not of rictor, causes metabolic changes and results in muscle dystrophy. Cell Metab. 2008;8(5):411-424.

36. Sauer B, Henderson N. Site-specific DNA recombination in mammalian cells by the Cre recombinase of bacteriophage P1. Proc Natl Acad Sci U S A 1988;85(14):5166-5170.

37. Curatolo P, Maria BL. Tuberous sclerosis. Handb
Clin Neurol. 2013;111:323-331.

38. Kwiatkowski DJ, Zhang H, Bandura JL, Heiberger KM, Glogauer M, el-Hashemite N, Onda H. A mouse model of TSC1 reveals sexdependent lethality from liver hemangiomas, and up-regulation of p70S6 kinase activity in Tsc1 null cells. Hum Mol Genet. 2002; 11(5):525-34.

39. Morrow EM, Furukawa T, Cepko CL. Vertebrate photoreceptor cell development and disease. Trends Cell Biol. 1998;8(9):353-358.

40. Andrieu-Soler C, et al. Intravitreous injection of PLGA microspheres encapsulating GDNF promotes the survival of photoreceptors in the rd1/ rd1 mouse. Mol Vis. 2005;11:1002-1011.

41. Ames A 3rd. CNS energy metabolism as related to function. Brain Res Brain Res Rev. 2000; 34(1-2):42-68.

42. Young RW. The renewal of rod and cone outer segments in the rhesus monkey. JCell Biol. 1971;49(2):303-318.

43. Duvel K, et al. Activation of a metabolic gene regulatory network downstream of mTOR complex 1 . Mol Cell.2010;39(2):171-183.

44. Mathupala SP, Ko YH, Pedersen PL. Hexokinase II: cancer's double-edged sword acting as both facilitator and gatekeeper of malignancy when bound to mitochondria. Oncogene. 2006;25(34):4777-4786.

45. Iqbal MA, Gupta V, Gopinath P, Mazurek S, Bamezai RN. Pyruvate kinase M2 and cancer: an updated assessment. FEBS Lett. 2014;588(16):2685-2692.

46. Semenza GL. HIF-1: upstream and downstream of cancer metabolism. Curr Opin Genet Dev. 2010;20(1):51-56.

47. Parker RO, Crouch RK. Retinol dehydrogenases (RDHs) in the visual cycle. Exp Eye Res. 2010;91(6):788-792.

48. Wang JS, Kefalov VJ. The cone-specific visual cycle. Prog Retin Eye Res. 2011;30(2):115-128.

49. Earnshaw WC, Martins LM, Kaufmann SH. Mammalian caspases: structure, activation, substrates, and functions during apoptosis. Annu Rev Biochem. 1999;68:383-424.

50. Choo AY, et al. Glucose addiction of TSC null cells is caused by failed mTORC1-dependent balancing of metabolic demand with supply. Mol Cell. 2010;38(4):487-499.

51. Guenther GG, et al. Loss of TSC2 confers resistance to ceramide and nutrient deprivation. Oncogene. 2014;33(14):1776-1787.

52. Punzo C, Xiong W, Cepko CL. Loss of daylight vision in retinal degeneration: are oxidative stress and metabolic dysregulation to blame? J Biol Chem. 2012;287(3):1642-1648.

53. Murakami Y, et al. Receptor interacting protein kinase mediates necrotic cone but not rod cell death in a mouse model of inherited degeneration. Proc Natl Acad Sci US A. 2012;109(36):14598-14603

54. Miller JW. Age-related macular degeneration revisited - piecing the puzzle: the LXIX Edward Jackson memorial lecture. Am JOphthalmol. 2013;155(1):1-35 e13.

55. Madisen L, et al. A robust and high-throughput Cre reporting and characterization system for the whole mouse brain. Nat Neurosci. 
2010;13(1):133-140.

56. Mattapallil MJ, et al. The Rd8 mutation of the Crb1 gene is present in vendor lines of C57BL/6N mice and embryonic stem cells, and confounds ocular induced mutant phenotypes. Invest Ophthalmol Vis Sci. 2012;53(6):2921-2927.

57. Venkatesh A, Ma S, Langellotto F, Gao G, Punzo
C. Retinal gene delivery by rAAV and DNA electroporation. Curr Protoc Microbiol. 2013;

Chapter 14:Unit 14D.4.

58. Zinchuk V, Wu Y, Grossenbacher-Zinchuk O, Stefani E. Quantifying spatial correlations of fluorescent markers using enhanced background reduction with protein proximity index and correlation coefficient estimations. Nat Protoc. 2011;6(10):1554-1567.

59. Ortin-Martinez A, et al. Number and distribution of mouse retinal cone photoreceptors: differences between an albino (Swiss) and a pigmented (C57/BL6) strain. PLoS One. 2014;9(7):e102392. 